\title{
Filmes e revestimentos comestíveis à base de polissacarídeos para aplicações alimentares
}

\author{
Miguel Ângelo Cerqueira \\ International Iberian Nanotechnology Laboratory, Av. Mestre José Veiga s/n, 4715-330, Braga, Portugal \\ miguel.cerqueira@inl.int
}

\begin{abstract}
Edible films and coatings based on polysaccharides for food applications - Films and edible coatings can be used with different functionalities. They have been used in the food industry, mainly in food preservation and as carriers of functional compounds, and in packaging functionalization. Its application is related to its ability to act as a barrier to gases, such as water vapor, oxygen and ethylene; with the possibility of adding to its matrix bioactive compounds that perform a certain function in the food or when ingested by the consumer; and by the possibility of wrapping food and thus enable the individual packaging thereof. Of all the compounds used, polysaccharides are the most exploited due to the large number of possible applications and great versatility. The main compounds used in the production of films and edible coatings, being given high relevance to those that use polysaccharides, are presented in this article. In addition, some examples of film and coating production methods and possible applications are given, emphasizing the layer-by-layer technique as a way of producing coatings and nanostructured films. The perspectives on the use of edible coatings and films and the main topics that will be addressed in the future are also discussed.
\end{abstract}

$\mathrm{O}_{\mathrm{s}}$ filmes e revestimentos comestíveis podem ser usados com diferentes funções. Na indústria alimentar eles têm sido usados principalmente na conservação de alimentos, como veículos para o transporte de compostos funcionais e na funcionalização de embalagens. A sua aplicação está relacionada com a sua capacidade de funcionar como uma barreira para gases, tais como vapor de água, oxigénio e etileno, com a possibilidade de incorporar na sua matriz compostos bioativos que desempenham uma determinada função no alimento ou quando ingeridos pelo consumidor; e pela possibilidade de envolver alimentos e assim possibilitar a embalagem individual dos mesmos. De todos os compostos utilizados, os polissacarídeos são os mais explorados devido ao grande número de possíveis aplicações, apresentando também uma grande versatilidade. Neste artigo são apresentados os principais compostos usados na produção de filmes e revestimentos comestíveis, sendo dada maior relevância aos que usam polissacarídeos como principal meio de produção. Além disso, são dados alguns exemplos de métodos de produção de filmes e revestimentos e possíveis aplicações, enfatizando a técnica camada-por-camada como uma forma de produzir revestimentos e filmes nanoestruturados. No final são apresentadas as perspetivas sobre o uso de revestimentos e filmes comestíveis e os principais tópicos que estarão em discussão no futuro.

\section{Introdução}

Os filmes e revestimentos comestíveis reapareceram nos últimos 20 anos devido às suas possíveis aplicações na indústria alimentar, onde estes foram apresentados como uma forma de garantir a qualidade dos alimentos e como uma estratégia no desenvolvimento de produtos inovadores. Estas aplicações vêm sendo aprimoradas através de novas propostas científicas e tecnológicas como resposta ao crescente interesse demonstrado pela indústria. Outro fator que também tem contribuído para o seu desenvolvimento é a procura, por parte dos consumidores, de soluções mais naturais e amigas do ambiente. São vários os compostos comestíveis que podem ser usados para produzir filmes e revestimentos, destacando-se os polissacarídeos, as proteínas e os lípidos. Os polissacarídeos, nomeadamente os derivados de celulose, quitosana, alginato, galactomananas, hemicelulose e amido são os mais usados pois são os que apresentam propriedades mais interessantes para aplicações alimentares. Os compostos para serem considerados comestíveis devem ser aprovados como ingredientes alimentares ou aditivos, ou devem estar no grupo de compostos que podem ser adicionados, e ingeridos, a produtos alimentares. A legislação atual é clara quanto aos ingredientes e aditivos que podem ser in- geridos com alimentos. No caso de filmes e revestimentos comestíveis esta legislação deve ser analisada caso-a-caso, de acordo com os alimentos onde se destinam a ser utilizados. Estes filmes ou revestimentos devem manter a sua edibilidade após o processamento e a aplicação, garantindo assim a denominação de "Geralmente Reconhecido como Seguro" (GRAS).

Recentemente, o uso de novos compostos, tais como polissacarídeos de novas fontes naturais e modificados quimicamente; a mistura de diferentes compostos (formação de compósitos); e o uso da nanotecnologia (nanopartículas e nanolaminados) levaram novas funções aos filmes e revestimentos e, portanto, novas oportunidades para a indústria alimentar. Essas oportunidades são promovidas pela: a) procura por compostos de base biológica (como polissacarídeos) que possam servir como alternativas aos compostos derivados de petróleo comumente usados em embalagens; b) necessidade de diminuir o uso de conservantes sintéticos em alimentos; c) busca por novas formas de evitar a migração de ingredientes nos alimentos, por exemplo quando se juntam alimentos sólidos e líquidos e não se pretende uma mistura imediata dos ingredientes; e d) individualização de alimentos, que permita ter alimentos ou ingredientes prontos a usar pelo consumidor final (ex.: em unidoses). 
Outra aplicação dos filmes e revestimentos comestíveis em alimentos envolve o transporte e libertação de compostos bioativos. Os compostos bioativos podem ser incorporados diretamente na matriz ou através de estruturas de encapsulação (microcápsulas ou nanoemulsões) que ficam aprisionadas e que permitem que os filmes e revestimentos sirvam de veículo e como uma forma de controlar a libertação dos compostos bioativos para os alimentos. Dependendo dos compostos usados é, também, possível controlar a sua libertação recorrendo a diferentes estímulos externos (compostos sensíveis ao $\mathrm{pH}$ e/ou à temperatura), dando uma nova funcionalidade ("inteligente”) aos filmes e revestimentos [1].

Neste artigo são apresentados os principais compostos usados na produção de filmes e revestimentos comestíveis, dando realce aos que usam polissacarídeos, e às possíveis aplicações. São também apresentados exemplos de métodos de produção de filmes e de revestimentos, dando ênfase à técnica camada-por-camada.

\section{Compostos}

Os compostos utilizados para produzir revestimentos ou filmes comestíveis devem atender a dois requisitos principais: i) devem ser considerados comestíveis e ii) devem ter a capacidade de formar um revestimento ou filme contínuo. Dos compostos com estas características destacam-se os polissacarídeos, as proteínas e as ceras. Este grupo de compostos pode ser dividido em diferentes categorias de acordo com o método de obtenção e origem, tais como: a) compostos diretamente obtidos de biomassa ou de outras fontes naturais (por exemplo, de fontes marinhas ou de animais); b) compostos produzidos por microrganismos; e c) compostos produzidos por síntese química. Destes materiais, os polissacarídeos são talvez os mais utilizados na produção de revestimentos e filmes comestíveis, tendo como grande vantagem o facto de serem já amplamente aplicados na indústria alimentar, como agentes emulsionantes, espumantes e gelificantes. Os principais fatores que diferenciam os polissacarídeos das proteínas são o facto de estes não apresentarem alergenicidade, de possuírem grande estabilidade ao pH (com a exceção de alguns tipos de quitosana), e de apresentarem grande estabilidade em solução.

São vários os fatores intrínsecos que podem modificar as propriedades e o comportamento final dos filmes e revestimentos. No caso dos polissacarídeos, esses fatores são a estrutura molecular, a presença de grupos funcionais, o peso molecular (PM) e a carga. Na Tabela 1 apresentam-se os polissacarídeos mais utilizados na produção de filmes e revestimentos comestíveis, a sua origem e algumas das suas principais características. Os polissacarídeos podem, também, ser usados em combinação com proteínas, ceras e/ou lípidos. As misturas resultantes podem ajudar na funcionalização e melhoria das propriedades dos filmes e revestimentos. As ceras, que foram aplicadas durante muitos anos como revestimento de frutas, têm sido utilizadas em combinação com polissacarídeos com o objetivo de melhorar as suas capacidades de barreira ao vapor de água bem como as suas propriedades mecânicas [2].

Na maioria dos casos, a formação de filmes e revestimentos requer a presença de um plasticizante. O tipo de plasticizante utilizado dependerá dos principais materiais utilizados para a produção dos filmes e revestimentos. No caso de compostos hidrofílicos, como a maioria dos polissacarídeos, a água é um dos plasticizantes mais efetivos e é a sua presença na matriz que influencia as propriedades dos filmes e revestimentos. Normalmente, os filmes à base de polissacarídeos sem plasticizante apresentam uma estrutura rígida e frágil. Os plasticizantes mais utilizados são os polióis (por exemplo, glicerol e sorbitol) que, quando incorporados na matriz, interferem nas interações intermoleculares levando a uma maior flexibilidade e processabilidade dos filmes. Outros compostos que podem ser adicionados são os surfactantes, que são normalmente classificados de acordo com o seu balanço hidrofílico-lipofílico. Os surfactantes são adicionados para aumentar a estabilidade dos filmes e revestimentos produzidos por misturas de polissacarídeos e lípidos e/ou ceras, permitindo a formação de uma emulsão e aumentando assim a sua estabilidade. Os surfactantes também podem ser incorporados para reduzir a tensão superficial dos revestimentos e melhorar a sua capacidade de revestir a superfície de alimentos, isto é, aumentando a sua molhabilidade.

Os compostos utilizados têm grande influência no desempenho dos filmes e revestimentos, afetando as suas principais propriedades de barreira, mecânicas e óticas. A forma de aplicação (por exemplo, camada-por-camada) e a presença de outros compostos adicionados (antimicrobianos ou antioxidantes) também podem afetar as características dos filmes e revestimentos, e isso deve ser avaliado antes da sua utilização.

\section{Aplicações}

Os filmes e revestimentos comestíveis podem ser usados com diferentes propósitos. Atualmente as aplicações mais debatidas e estudadas são a sua utilização como embalagem e a funcionalização de embalagens. Antes de mais, é importante definir embalagem e de que forma podemos enquadrar os filmes e revestimentos neste grupo de materiais. Uma embalagem é usada para proteger os bens, facilitar a sua distribuição e informar o consumidor. No caso dos alimentos, essa propriedade é potenciada, uma vez que as embalagens podem ser usadas para garantir a preservação de produtos perecíveis, fornecer informações importantes aos consumidores e garantir a sua conveniência [3]. Os filmes e revestimentos comestíveis são capazes de proteger os alimentos e trazer conveniência ao consumidor e, portanto, podem ser enquadrados na definição de embalagem.

Na indústria alimentar, o metal, o vidro, o papel e o plástico são os materiais de embalagem mais utilizados, mas nos últimos anos tem-se verificado um aumento do uso de bioplásticos, nomeadamente na produção de embalagens à base de ácido poli(láctico) e de poli(hidroxibutanoato). Considerando a origem e biodegradabilidade dos bioplásticos, e atendendo às preocupações ambientais, estes representam uma forma de reduzir o uso de materiais derivados do petróleo e de diminuir a produção de resíduos. Neste contexto, os filmes e revestimentos comestíveis podem ajudar a reduzir o uso de materiais não-biodegradáveis, uma vez que são baseados em compostos de grau alimentar, que podem ser ingeridos, e são totalmente biodegradáveis. 
Tabela 1 - Exemplos de polissacarídeos utilizados na produção de filmes e revestimentos.

\begin{tabular}{|c|c|c|}
\hline Polissacarídeo & Fonte & Características \\
\hline Pectina & Vegetais & $\begin{array}{l}\text { Solúvel em água (para total solubilização poderá ser necessá- } \\
\text { ria temperatura elevada). Capacidade de formação de filmes } \\
\text { e revestimentos em concentrações de } 0,5 \text { a } 2 \%(\mathrm{p} / \mathrm{v}) \text {, depen- } \\
\text { dendo do grau de esterificação e PM. Carregada negativa- } \\
\text { mente a pH >3,5 e sensível a variações de } \mathrm{pH} \text {. }\end{array}$ \\
\hline
\end{tabular}

Galactomananas $\quad$ Vegetais

Solúvel em água (para total solubilização poderá ser necessária temperatura elevada). Capacidade de formação de filmes e revestimentos em concentrações de 0,5 a 3\% (p/v), dependendo da razão manose/galactose e PM. Neutra.

\begin{tabular}{lll}
\hline Carboximetilcelulose & $\begin{array}{l}\text { Celulose obtida } \\
\text { de vegetais ou } \\
\text { produzida por mi- } \\
\text { crorganismos }\end{array}$ & $\begin{array}{l}\text { Solúvel em água. Capacidade de formação de filmes e reves- } \\
\text { timentos em concentrações de 0,5 a 3\% (p/v), dependendo do } \\
\text { grau de substituição e PM. Carregada negativamente. }\end{array}$ \\
\hline Etilcelulose & $\begin{array}{l}\text { Celulose obtida } \\
\text { de vegetais ou } \\
\text { produzida por mi- } \\
\text { crorganismos }\end{array}$ & $\begin{array}{l}\text { Solúvel em solventes orgânicos. } \\
\text { Capacidade de formação de filmes e revestimentos em con- } \\
\text { centrações de 0,5 a 5\% (p/v), dependendo do grau de substi- } \\
\text { tuição e PM. Neutra. }\end{array}$ \\
\hline Alginato & $\begin{array}{l}\text { Algas ou produ- } \\
\text { zida por micror- } \\
\text { ganismos }\end{array}$ & $\begin{array}{l}\text { Solúvel em água. Capacidade de formação de filmes e reves- } \\
\text { timentos em concentrações de 0,5 a 3\% (p/v), dependendo } \\
\text { da razão de ácido manurónico/ácido galurónico e PM. Ca- } \\
\text { pacidade de reticulação na presença de iões multivalentes. } \\
\text { Carregado negativamente. }\end{array}$ \\
& $\begin{array}{l}\text { Solúvel em meio ácido (normalmente solúvel a pH <4,5). } \\
\text { Capacidade de formação de filme e revestimento em concen- } \\
\text { trações de 0,5 a 3\% (p/v), dependendo do grau de acetilação } \\
\text { e PM. Apresenta atividade antimicrobiana. Carregada posi- } \\
\text { tivamente. }\end{array}$ \\
\hline ganismos pou micror- &
\end{tabular}

A aplicação de um revestimento ou filme comestível num produto alimentar depende da função pretendida (barreira a gases ou transporte de compostos bioativos), do tipo de alimento (produtos lácteos, frutas, café solúvel, etc.) e das condições de armazenamento (temperatura e humidade relativa, por exemplo).

\section{Revestimento}

O revestimento é uma solução que é aplicada diretamente a uma superfície e que, após a secagem, forma um filme fino, executando assim a função desejada. Ele pode ser utilizado diretamente na superfície do alimento ou aplicado em materiais de embalagem já pré-formados. Os métodos de aplicação dependem da superfície onde o revestimento vai ser aplicado e da constituição dos revestimentos, mas os métodos mais utilizados são a imersão e a pulverização. Outra técnica de aplicação que tem sido explorada nos últimos anos em aplicações alimentares é a técnica camada-por-camada. Esta técnica envolve a adsorção de moléculas carregadas, em solução aquosa, a um suporte, em que a adsorção sequencial de materiais com cargas opostas (por exemplo, polissacarídeos, nanopartículas) resulta na formação de uma estrutura multicamada na qual cada camada pode atingir alguns nanómetros [4, 5]. A formação das multicamadas não envolve a formação de li- gações covalentes, mas podem estar envolvidas interações intermoleculares (ligações de hidrogénio, por exemplo). Os tipos de compostos usados para produzir cada camada, o número total de camadas incorporadas, a sua sequência e as condições usadas para preparar cada camada determinarão as propriedades e a funcionalidade do revestimento multicamada produzido [6]. A versatilidade e o sucesso da técnica camada-por-camada baseia-se na facilidade e adaptabilidade do processo, bem como na capacidade de usar várias geometrias como suporte, podendo ser utilizada em superfícies planas [7,8], mas também em cápsulas e estruturas tubulares [9-12]. As principais limitações da técnica são o tempo necessário durante a aplicação e a forma de aplicação, mas estas têm sido ultrapassadas nos últimos anos, verificando-se grandes avanços com o desenvolvimento de dispositivos capazes de reduzir tanto o tempo (por exemplo, usando sistemas de pulverização) como o trabalho associado à sua aplicação (por exemplo, através de métodos automatizados de imersão e pulverização) $[13,14]$.

A aplicação direta de revestimentos em alimentos permite aumentar o tempo de prateleira de vários produtos alimentares, onde a aplicação de uma única camada [14-17] ou de multicamadas tem mostrado excelentes resultados em frutas, vegetais, queijos e peixe [18-21]. A Figura 1, obtida por microscopia eletrónica de varrimento, apresenta 

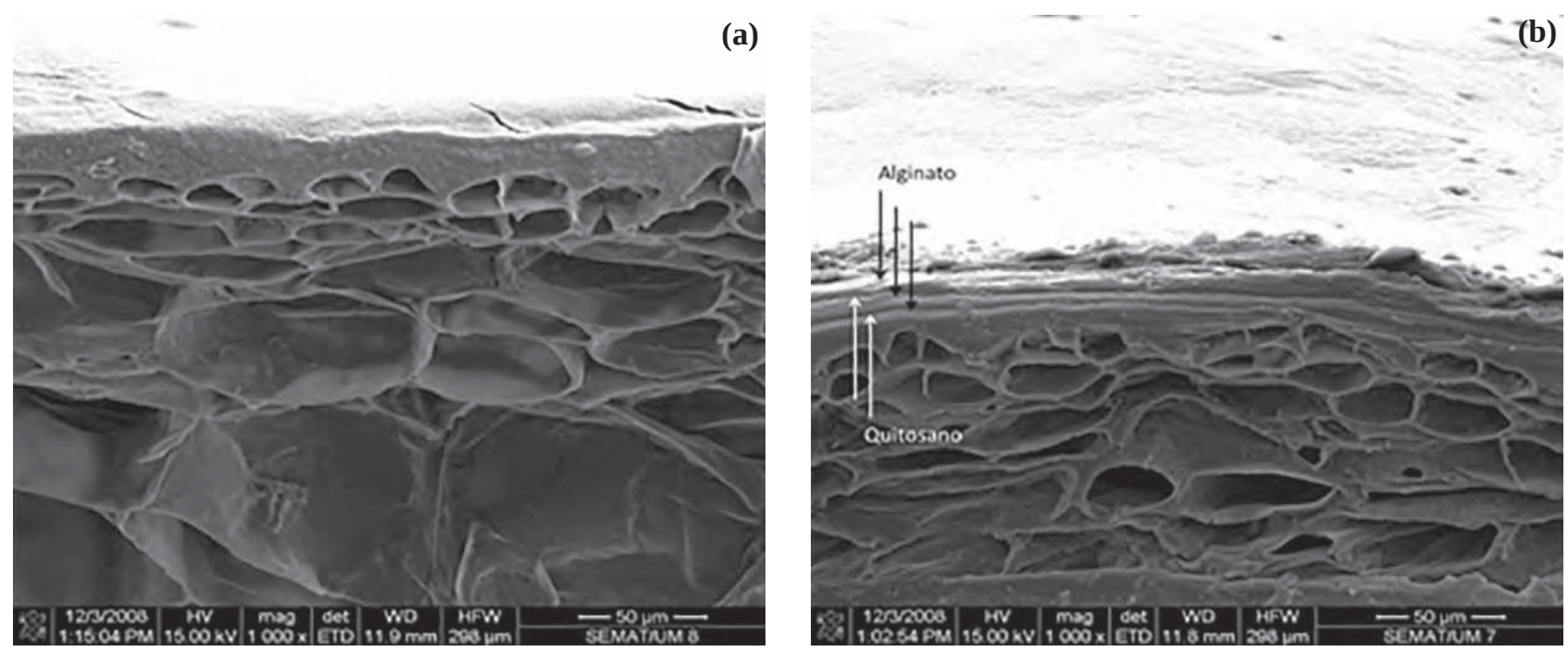

Figura 1 - (a) Imagem de microscopia eletrónica de varrimento da superfície da manga e (b) de revestimento nanolaminado na superfície da manga (alginato/quitosana/alginato/quitosana/alginato) [22]. Copyright 2015, com permissão da Springer.

a superfície de uma manga com a aplicação de um revestimento multicamada, isto é, camadas sucessivas de alginato e de quitosana.

Os revestimentos têm a capacidade de reduzir a perda de massa, os processos de transferência gasosa, assim como alguns fenómenos de amadurecimento das frutas. Além da sua atuação como uma barreira a gases, também podem ter uma função antimicrobiana ou bacteriostática. Este efeito é potenciado quando são usados compostos antimicrobianos, tais como péptidos bioativos, óleos essenciais, ou outros compostos que permitam manter o seu carácter comestível e ao mesmo tempo aumentar a sua funcionalidade. Um exemplo interessante envolve o uso de nisina, um péptido bioativo, num revestimento à base de polissacarídeos, que foi aplicado em queijo fresco com o objetivo de diminuir o crescimento de Listeria monocytogenes durante o armazenamento [23]. Outro exemplo envolve o uso do óleo essencial da laranja em revestimentos à base de alginato, o qual foi usado para diminuir o crescimento de fungos e leveduras em framboesas vermelhas durante o armazenamento [24].

No caso da aplicação em materiais de embalagem, estes revestimentos são usados para melhorar as suas propriedades de barreira ou de transporte de compostos bioativos que podem ter um efeito antimicrobiano e/ou antioxidante nos alimentos. Um dos processos mais simples, amplamente utilizado à escala laboratorial, é a aplicação dos revestimentos na superfície do produto de interesse e posterior secagem através da evaporação do solvente, resultando na formação de um filme. Esta técnica permite o uso de revestimentos comestíveis como veículos de compostos antimicrobianos e antioxidantes, visando a funcionalização de embalagens convencionais. Por exemplo, a combinação de quitosana com óleos essenciais foi usada para revestir filmes de polipropileno. As embalagens desenvolvidas foram testadas contra Listeria monocytogenes, Staphylococcus aureus, e Escherichia coli e verificou-se que as embalagens desenvolvidas apresentavam propriedades antimicrobianas contra os microrganismos testados [25].

A técnica camada-por-camada também pode ser usada para modificar as propriedades de barreira de filmes de embalagens comerciais [26]. Já foram testados vários compostos mas apenas os que apresentam o estatuto de GRAS e que estão aprovados como Materiais em Contacto com Alimentos devem ser usados para aplicações em embalagens de alimentos. Nesse sentido, vários compostos de base biológica e comestíveis têm mostrado características interessantes para o desenvolvimento de estruturas multicamadas utilizando a técnica de camada-por-camada.

Uma das propostas mais interessantes é a formação de uma estrutura tipo "parede de tijolos" à nanoescala que combina a formação de multicamadas com a incorporação de nanopartículas, a fim de diminuir a permeabilidade aos gases. Esta combinação leva a um aumento da tortuosidade dos filmes, o que faz com que as moléculas de oxigénio tenham de percorrer um maior caminho para se difundir pelo filme, diminuindo assim a sua permeabilidade [27]. Esta proposta foi apresentada utilizando quitosana [28] ou k-carragenano [29] com nanopartículas de argila em poli(tereftalato de etileno) (PET) e mostrou-se capaz de reduzir a permeabilidade ao oxigénio.

O método camada-por-camada também permite a incorporação de compostos bioativos, tais como óleos essências e seus derivados. Estes apresentam propriedades antimicrobianas [30] mas possuem algumas desvantagens tais como a baixa solubilidade em água e elevada volatilização. É por isso necessário encontrar novas estratégias para a sua incorporação em embalagens, nomeadamente a encapsulação. Recentemente, foram testados dois sistemas de multicamadas para o desenvolvimento de embalagens ativas [31]. Neste caso, o carvacrol foi adicionado a um sistema multicamada, encapsulado em nanocápsulas de zeína ou emulsionado numa solução de quitosana. Estes dois sistemas de cinco camadas foram desenvolvidos num filme de poli(tereftalato de etileno) usando as seguintes arquiteturas: 1) alginato/nanocápsulas de zeína/alginato/ nanocápsulas de zeína/alginato, ou 2) alginato/emulsão de quitosana/alginato/emulsão de quitosana/alginato. Estes filmes bioativos foram testados contra o fungo Alternaria sp. e verificou-se que o filme multicamada com alginato/ nanocápsulas de zeína/alginato/nanocápsulas de zeína/alginato apresentou os melhores resultados e a maior ativi- 
dade antifúngica (diminuição de cerca de 30\% de unidades formadoras de colónias totais relativamente ao controlo).

A técnica camada-por-camada também pode ser uma excelente abordagem para o desenvolvimento de sistemas de embalagens inteligentes. Vários trabalhos têm mostrado a possibilidade de usar esta técnica para o desenvolvimento de sistemas que respondam à modificação do pH [32,33] e/ ou adsorção de eletrólitos [34], ou mudanças de temperatura [35], tornando-os atrativos para utilização em sistemas de embalagens. No entanto, ainda há alguns desafios que devem ser superados, incluindo a melhoria das propriedades mecânicas dos materiais desenvolvidos, otimização das condições de produção, e a exploração de novos mecanismos de resposta.

\section{Filmes}

Os filmes podem ser produzidos pelo "processo húmido” ou pelo "processo seco”, e podem apresentar uma espessura que pode variar de apenas alguns micrómetros até algumas centenas de micrómetros [36]. O processo escolhido dependerá dos compostos utilizados, assim como da sua aplicação. O processo mais comum é o "processo húmido", onde os compostos são dissolvidos ou dispersos num solvente originando uma mistura homogénea que é colocada num molde. O filme é formado por evaporação do solvente. Normalmente é utilizada uma solução aquosa, mas em alguns casos (por exemplo, etilcelulose) é necessário um outro solvente, como o etanol, para melhorar a solubilização. Nestes casos, os solventes devem estar aprovados para aplicações alimentares (grau alimentar), uma vez que, apesar da sua evaporação, podem permanecer alguns resíduos na matriz do filme e posteriormente migrarem para os alimentos. O outro processo utilizado é o "processo seco", onde o comportamento térmico dos compostos é usado como meio de fluidização dos materiais (por exemplo, temperatura de fusão ou temperatura de transição) sem o uso de solventes, Este método é menos comum na produção de filmes à base de polissacarídeos.

Os filmes edíveis são desde há muito tempo usados para transportar nutrientes e compostos bioativos e já existem muitas aplicações com a sua utilização [37]. Por exemplo, a empresa Watson (USA) comercializa estes filmes edíveis com cafeína, nutrientes e outros compostos ativos [38]. No caso da conservação de alimentos, eles podem ser usados como uma embalagem, que funciona como um invólucro, que vai proteger o alimento e ao mesmo tempo trazer mais conveniência para o consumidor. Em termos de conveniência para o consumidor, alguns trabalhos mostraram a possibilidade de produzir filmes edíveis que podem ser usados para produzir unidoses e que depois de dispersos em água se solubilizam instantaneamente. Dois dos exemplos práticos são as doses individuais de café solúvel ou doses individuais de arroz, que podem ser colocados diretamente no local onde vão ser consumidos ou cozinhados, respetivamente. Outra possibilidade é formar um filme que será ingerido diretamente com o alimento, sendo este menos usado mas já muito explorado por algumas empresas [39].

\section{Perspetivas futuras}

É espectável que os filmes e revestimentos comestíveis continuem o seu caminho para a maturidade industrial e comercial, existindo já várias empresas a explorar a sua utilização. No entanto, existem alguns desafios que estão relacionados com as suas propriedades de baixo desempenho (ex.: elevada permeabilidade a vapor de água) e alto custo quando comparados com materiais à base de petróleo. Atualmente são várias as abordagens para melhorar as propriedades dos filmes e revestimentos e reduzir o seu custo. No primeiro caso, a utilização de nanomateriais edíveis (nanocristais de celulose) e a utilização de técnicas inovadoras (por exemplo, processo eletro-hidrodinâmico e atomização vibracional) podem aumentar a aplicabilidade e funcionalidade e melhorar as propriedades destes materiais. Relativamente ao custo, dá-se preferência ao uso de compostos provenientes de fontes de baixo custo (subprodutos da indústria, como amido ou pectina) e que mantenham as suas propriedades mesmo que usados em menores quantidades. Apesar das suas propriedades únicas, os filmes e revestimentos à base de polissacarídeos dificilmente irão eliminar por completo as embalagens à base de derivados de petróleo, mas poderão ajudar a funcionalizar estes materiais e diminuir a sua utilização. Nesse sentido, a sua aplicação como um material comestível, sendo diferenciador dos demais, deve ser considerada de acordo com a aplicação, e a sua viabilidade comercial deve ser bem estudada. Um dos fatores diferenciadores que deve ser explorado é o seu uso como veículo de compostos funcionais, visando não só a conservação dos alimentos mas também a ingestão de ingredientes funcionais (por exemplo, vitaminas e probióticos) em combinação com alimentos por parte do consumidor. A sua utilização poderá assim aumentar o número de aplicações possíveis e melhorar a biodisponibilidade dos ingredientes funcionais.

\section{Referências}

[1] M.A. Cerqueira, J.A.C. Teixeira, A.A. Vicente, Edible packaging today, in Edible Food Packaging: Materials and Processing Technologies, CRC Press, 2016, pp. 1-8.

[2] J.A. Aguirre-Joya, B. Álvarez, J.M. Ventura, J.O. GarcíaGalindo, M.A. De León-Zapata, R. Rojas, S. Saucedo, C.N. Aguilar, Edible coatings and films from lipids, waxes, and resins, in Edible Food Packaging: Materials and Processing Technologies, CRC Press, 2016, pp. 121-152.

[3] P. Zepf, Appendix B: Glossary of Packaging Terminology and Definitions, in K.L. Yam (Ed.), Wiley Encycl. Packag. Technol., John Wiley \& Sons, Inc., 2010, pp. 1287-1304.

[4] G. Decher, J.-D. Hong, Makromol. Chemie Macromol. Symp. 46 (1991) 321-327.

[5] G. Decher, Science 277 (1997) 1232-1237.

[6] C. Porcel, P. Lavalle, V. Ball, G. Decher, B. Senger, J.C. Voegel, P. Schaaf, Langmuir 22 (2006) 4376-4383.

[7] C. Liu, X. Lei, L. Wang, J. Jia, X. Liang, X. Zhao, H. Zhu, Chem. Eng. J. 327 (2017) 60-70.

[8] M.G. Carneiro-da-Cunha, M.A. Cerqueira, B.W.S. Souza, S. Carvalho, M.A.C. Quintas, J.A. Teixeira, A.A. Vicente, Carbohydr. Polym. 82 (2010) 153-159.

[9] B. Tugba Camic, F. Oytun, M. Hasan Aslan, H. Jeong Shin, H. Choi, F. Basarir, J. Colloid Interface Sci. 505 (2017) 79-86.

[10] A.C. Pinheiro, A.I. Bourbon, M.A. Cerqueira, É. Maricato, C. Nunes, M.A. Coimbra, A.A. Vicente, Carbohydr. Polym. 115 (2015) 1-9. 
[11] M.C. Rivera, A.C. Pinheiro, A.I. Bourbon, M.A. Cerqueira, A.A. Vicente, Int. J. Biol. Macromol. 79 (2015) 95-102.

[12] J.J. Richardson, M. Björnmalm, F. Caruso, Science 348 (2015) aaa2491.

[13] J.J. Richardson, J. Cui, M. Björnmalm, J.A. Braunger, H. Ejima, F. Caruso, Chem. Rev. 116 (2016) 14828-14867.

[14] M.A. Cerqueira, A.M. Lima, B.W.S. Souza, J.A. Teixeira, R.A. Moreira, A.A. Vicente, J. Agric. Food Chem. 57 (2009) 1456-1462.

[15] B.W.S. Souza, M.A. Cerqueira, H.A. Ruiz, J.T. Martins, A. Casariego, J.A. Teixeira, A.A. Vicente, J. Agric. Food Chem. 58 (2010) 11456-11462.

[16] M.A. Cerqueira, M.J. Sousa-Gallagher, I. Macedo, R. Rodriguez-Aguilera, B.W.S. Souza, J.A. Teixeira, A.A. Vicente, J. Food Eng. 97 (2010) 87-94.

[17] J.T. Martins, M.A. Cerqueira, B.W.S. Souza, M.D.O. Carmo Avides, A.A. Vicente, J. Agric. Food Chem. 58 (2010) 1884-1891.

[18] B.G.S. Medeiros, M.P. Souza, A.C. Pinheiro, A.I. Bourbon, M.A. Cerqueira, A.A. Vicente, M.G. Carneiro-da-Cunha, Food Bioprocess Technol. 7 (2014) 1088-1098.

[19] B.G. de S. Medeiros, A.C. Pinheiro, J.A. Teixeira, A.A. Vicente, M.G. Carneiro-da-Cunha, Food Bioprocess Technol. 5 (2012) 2435-2445.

[20] B.G. Bartolomeu, A.C. Pinheiro, M.G. Carneiro-Da-Cunha, A.A. Vicente, J. Food Eng. 110 (2012) 457-464.

[21] M.P. Souza, A.F.M. Vaz, M.A. Cerqueira, J.A. Texeira, A.A. Vicente, M.G. Carneiro-da-Cunha, Food Bioprocess Technol. 8 (2014) 647-654.

[22] M.L. Flores-López, M.A. Cerqueira, D.J. de Rodríguez, A.A. Vicente, Food Eng. Rev. 8 (2016) 292-305.

[23] J.T. Martins, M.A. Cerqueira, B.W.S. Souza, M.D.O. Carmo Avides, A.A. Vicente, J. Agric. Food Chem. 58 (2010) 1884-1891.

[24] M. de S. Gomes, M. das G. Cardoso, A.C.G. Guimarães, A.C. Guerreiro, C.M.L. Gago, E.V. de B. Vilas Boas, C.M.B. Dias, A.C.C. Manhita, M.L. Faleiro, M.G.C.
Miguel, M.D.C. Antunes, J. Sci. Food Agric. 97 (2017) 929-938.

[25] E. Torlak, M. Nizamlioğlu, J. Plast. Film Sheeting 27 (2011) 235-248.

[26] W.S. Jang, I. Rawson, J.C. Grunlan, Thin Solid Films 516 (2008) 4819-4825.

[27] C. Silvestre, D. Duraccio, S. Cimmino, Prog. Polym. Sci. 36 (2011) 1766-1782.

[28] G. Laufer, C. Kirkland, A.A. Cain, J.C. Grunlan, ACS Appl. Mater. Interfaces 4 (2012) 1643-1649.

[29] G. Laufer, C. Kirkland, A.A. Cain, J.C. Grunlan, Carbohydr. Polym. 95 (2013) 299-302.

[30] K.A. Hammer, C.F. Carson, T. V. Riley, J. Appl. Microbiol. 86 (1999) 985-990.

[31] M.J. Fabra, M.L. Flores-López, M.A. Cerqueira, D.J. de Rodriguez, J.M. Lagaron, A.A. Vicente, Food Bioprocess Technol. 9 (2016) 471-480.

[32] Y. Lu, Y. Wu, J. Liang, M.R. Libera, S.A. Sukhishvili, Biomaterials 45 (2015) 64-71.

[33] I. Zhuk, F. Jariwala, A.B. Attygalle, Y. Wu, M.R. Libera, S.A. Sukhishvili, ACS Nano 8 (2014) 7733-7745.

[34] E. Kharlampieva, S.A. Sukhishvili, Langmuir 20 (2004) 9677-9685.

[35] Z. Zhao, L. Yin, G. Yuan, L. Wang, Langmuir 28 (2012) 2704-2709.

[36] G.L. Robertson, Edible, biobased and biodegradable food packaging materials, in Food Packaging: Principles and Practice, 3rd ed., CRC Press, Boca Raton, Florida, 2012, pp. 49-90.

[37] A.F. Borges, C. Silva, J.F.J. Coelho, S. Simões, J. Control. Release 206 (2015) 1-19.

[38] Watson (2017). http://www.watson-inc.com/our-capabilities/film-technology/edible-films/ (acedido em 13 de outubro de 2017).

[39] M. Nieto, Edible film and packaging using gum polysaccharides, in Edible Food Packaging: Materials and Processing Technologies, CRC Press, 2016, pp. 9-79.

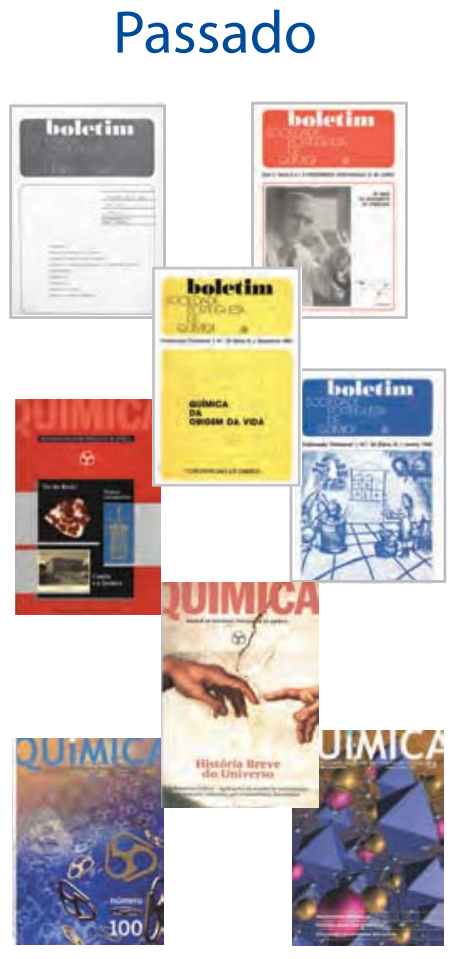

A Sociedade Portuguesa de Química (SPQ) foi fundada em Dezembro de 1911

Publica desde 1977 um boletim trimestral QUÍMICA.

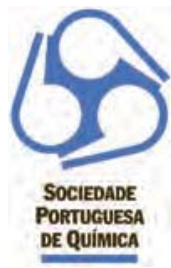

Presentemente está envolvida na publicação de treze revistas europeias de grande prestígio no âmbito da sociedade internacional ChemPubSoc Europe.

\section{Presente e Futuro}

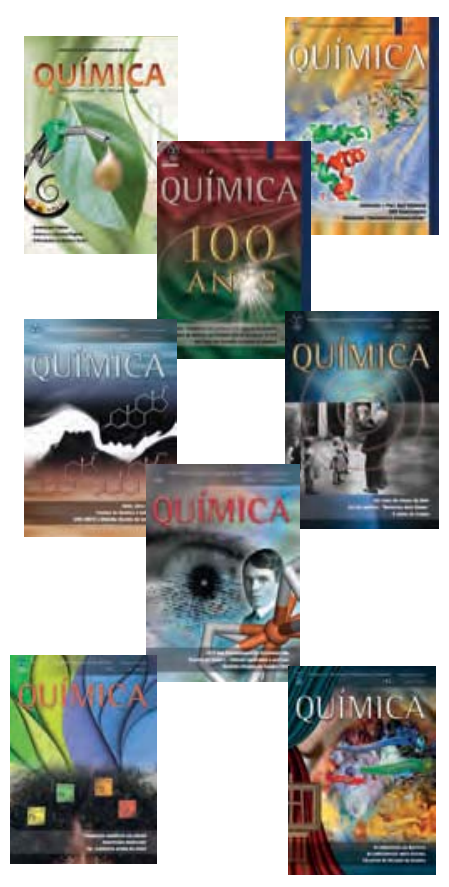

